\title{
Traduire
}

Une autre perspective sur r tr traduction

Revue française de la traduction

$216 \mid 2008$

Question de confiance

\section{La Théorie interprétative de la traduction}

\section{Freddie Plassard}

\section{(2) OpenEdition}

\section{Journals}

Édition électronique

URL : http://journals.openedition.org/traduire/995

DOI : 10.4000/traduire.995

ISSN : 2272-9992

\section{Éditeur}

Société française des traducteurs

\section{Édition imprimée}

Date de publication : 1 mars 2008

Pagination : 84-91

ISSN : 0395-773X

\section{Référence électronique}

Freddie Plassard, «La Théorie interprétative de la traduction », Traduire [En ligne], 216 | 2008, mis en ligne le 01 mars 2008, consulté le 23 septembre 2020. URL : http://journals.openedition.org/traduire/ 995 ; DOI : https://doi.org/10.4000/traduire.995 


\title{
La Théorie interprétative de la traduction
}

\author{
Freddie Plassard
}

\author{
F. Israël, M. Lederer (dir.) \\ Lettres modernes Minard, Cabiers Champollion 6 à 8 , \\ Paris, Caen, 2005 \\ Tome I : Genèse et développement (198 p.) \\ T. II : Convergences et mises en perspectives (300 p.) \\ T. III : De la formation... à la pratique professionnelle (184 p.)
}

L'ouvrage en trois tomes publié en hommage à $\mathrm{D}$. Seleskovitch, décédée le 17 avril 2001, retrace le parcours d'exception de l'interprète, enseignante et théoricienne qu'elle fut, à travers une multiplicité d'éclairages jetés sur son ouvre, la théorie interprétative de la traduction, et ses prolongements.

Si le tout premier article (A.-M. Widlund-Fantini) commence par poser des jalons biographiques qui semblent a posteriori avoir prédestiné Seleskovitch à la carrière qu' elle a connue - études en Allemagne, en ex-Yougoslavie, exercice de l'interprétation aux États-Unis - le premier tome distribue en quelque sorte les cartes, en déclinant les thèmes qui seront pleinement déployés dans les deux autres tomes, comme autant de points de focalisation, autorisant du reste une lecture transversale de l'ouvrage. On y décèle d'entrée de jeu le souci constant de situer la théorie interprétative par rapport à ses sources, exégètes voire contradicteurs, autrement dit d'éclairer, expliciter un intertexte théorique parfois resté implicite.

\section{Sources}

Dans le premier tome intitulé Genèse et développement se trouvent en effet exposées les origines de la théorie interprétative, comme jamais 
elles ne l'avaient été dans les précédentes publications de cette École. Clarté, repères précis, complets et commentés, de nature tant biographique que bibliographique et historique, sont au service d'une recontextualisation des événements et du climat intellectuel dans lequel s'est progressivement élaborée cette théorie.

S'il n'est plus nécessaire de souligner l'originalité de la pensée de D. Seleskovitch, il semble également aller de soi qu'elle ne pouvait, dans sa démarche de recherche, faire abstraction des écrits qui en étaient contemporains, soit pour les contrer, comme ce fut souvent le cas, soit pour s'en approprier les éléments éventuellement pertinents à une théorisation de la pratique de l'interprétation. Les articles de C. Laplace et de M. Lederer font la pleine lumière sur la genèse d'une pensée, comme si, par l'accès qui nous est donné à la bibliothèque de D. Seleskovitch, nous était aussi donnée la possibilité de comprendre, preuves à l'appui, notamment sous forme de citations, dans quel type de dialogue intérieur ou avec des ouvrages et des auteurs, avait pris forme la théorie interprétative.

La somme que représentent les trois volumes est aussi l'occasion de dresser un bilan des principaux acquis de la théorie interprétative, que «l'on peut aujourd'hui considérer comme une théorie générale du processus de la traduction, ce qui ne signifie nullement qu'elle est close une fois pour toutes... » (Lederer, p. 89) et qu'on peut formuler rapidement de la sorte : traduire, opération cognitive et non pas linguistique, n'a pas pour objet les langues, mais le sens appréhendé dans une situation de communication, « consciemment et délibérément construit par deux subjectivités (...) à l'interface de deux consciences » (Laplace, pp. 45-46). À partir de ce postulat de départ, la production théorique de D. Seleskovitch se poursuit dans deux directions : l'une à visée didactique, développée et illustrée dans le tome 3 de l'ouvrage, l'autre davantage axée sur la philosophie du langage, vaste terrain d'exploration pour une théorie qui n'a cessé d'évoluer depuis quarante ans et dont témoigne son ouverture à des positions et pratiques autres que celle qui lui a servi de point de départ, celle de l'interprétation. La part accordée à la traduction littéraire, à la traduction en langue 
des signes l'atteste. La méthodologie de recherche adoptée (t. I, p. 23 et sq.) est celle d'une démarche empirique et inductive, partant de la pratique pour extrapoler et dégager des axiomes. Elle implique de vérifier les dires des praticiens et de tenter de les expliquer, de façon à approfondir l'approche didactique et à permettre de théoriser sur le fonctionnement du langage, sans chercher à démontrer la connaissance des langues.

\section{«Cousinages intellectuels $(1)$ 》}

C'est toutefois par la mise en parallèle ou le dialogue avec d'autres théories, qu'elles soient traductologiques ou non, que l'objet de la théorie interprétative et les prises de position dont elle est caractéristique s'éclairent le mieux, par contraste. Ici, deux axes se dessinent de nouveau : d'une part les autres théories de la traduction existantes ou les critiques formulées à l'encontre de la théorie interprétative, et de l'autre les réflexions théoriques issues d'horizons tout différents.

Si M. Lederer, cofondatrice de la théorie interprétative, souligne que cette théorie véhicule des « principes qui dérangent et qui ont du mal à s'imposer », elle a également à cour de battre en brèche nombre d'idées reçues à son sujet, constatant qu'à ce jour et en dépit de toutes les critiques formulées, dans lesquelles entrent en proportion non négligeable les erreurs ou omissions de lecture, nul n'a été en mesure d'avancer une théorie générale qui viendrait s'y substituer ou la détrôner (p. 134). Se trouvent ainsi réfutés les arguments d'une spécificité de l'interprétation selon les langues, d'une absence de prise en compte de la forme en traduction - les nombreuses thèses sur la traduction littéraire soutenues dans ce cadre théorique et institutionnel étant là pour en témoigner - et celui de l'objectivité discutable du sens, la notion d'interprétation étant à entendre comme le fait de « comprendre objectivement le vouloir-dire de l'auteur à travers le dit du texte » (p. 122). On notera ici que la citation des propos parfois virulents

(1) Intitulé de l'article de J.-R. Ladmiral dans le tome I, p. 141. 
ou dépréciateurs tenus par les détracteurs montre a contrario la force acquise par la théorie en question, puisque le discours de contradiction peut lui-même être intégré au corpus des textes fondateurs, suffisamment conforté par les années de pratique, les corroborations diverses et les travaux qui la prolongent pour y accueillir la critique. La théorie interprétative ressort de cette confrontation comme constituant un corps théorique abouti, unifié et de portée universelle mais non clos.

Parmi les disciplines avec lesquelles le dialogue a pour ainsi dire été tenu de s'établir, figure la linguistique, longtemps synonyme de générativisme et de comparatisme dans les écrits de l'École de Paris. D'un côté les émules de Chomsky et les grammaires génératives déclinant les règles de composition ou de maniement du langage, de l'autre les adeptes de la comparaison des modes d'expression entre deux voire plusieurs langues. Si ces deux courants prévalaient dans le contexte de formulation initiale de la théorie interprétative, il semble que la situation ait singulièrement évolué. Les premières études linguistiques de la traduction trouvaient sans doute leurs limites, précise J.-R. Ladamiral, dans le fait que la traduction n'y était qu'un élément d'un dispositif de recherche et non l'objet à proprement parler de la discipline (t. I, p. 149). Aussi est-il amené à resituer la théorie interprétative dans ses « cousinages intellectuels », tant en amont, les sources possibles et attestées, qu'en aval, les prolongements possibles dont témoignent le tome II en déployant toutes les virtualités de sens et d'interprétation des thèses exposées dans les ouvrages qui constituent le noyau dur de cette théorie, mais aussi le tome III, sur le terrain de la pédagogie et de la pratique.

Théorie de portée universelle et générale, la théorie interprétative de la traduction se prête au fil des relectures diachroniques, à de saisissants rapprochements tant avec la sémantique interprétative (F. Rastier), le dialogisme (Bakhtine), la sémiotique (U. Eco), l'intertextualité, notion élaborée dans le cadre de la critique littéraire des années 1970 ou les modèles cognitifs de la compréhension à la lecture. Il est dès lors quasi impossible de vouloir rendre compte de toutes les contributions et c'est à la notion d'interprétation telle qu'elle se dégage 
de l'analyse comparative qui en est faite par G. Roux-Faucard que nous nous intéresserons.

Par confrontation avec la sémantique interprétative de F. Rastier, la notion et ses contours se détachent progressivement du contexte verbal où elle s'insère, pour parvenir à une clarté quasi définitoire. G. Roux-Faucard s'attache à démontrer, telle un détective menant l'enquête au plus près des textes et des indices énonciatifs, en quoi deux approches que tout a priori devrait diviser, convergent en réalité, sous réserve de remonter le fil qui permet de tisser l'isotopie de l'interprétation dans des corpus théoriques de prime abord fort distants. Il en résulte que :

La traduction par équivalence ne suppose pas la conservation du sémème, mais la conservation du sens, c'est-à-dire uniquement celle de sèmes organisés selon certaines isotopies. [...] Si l'on ose penser jusqu'au point où elle nous invite à le faire, la traduction « interprétative » n'aurait donc plus à prendre en compte que deux types d'unités de sens : les sèmes et les isotopies (p. 80).

Ce rapprochement amène à progressivement mieux cerner les notions de sens et d'unité de sens, en invitant à se focaliser sur les « sèmes et isotopies », autrement dit les éléments pertinents permettant de distinguer sémantiquement deux termes d'une même série(2) à l'échelle microtextuelle d'une part, et les ensembles « de significations caractérisées par leur appartenance à un même niveau de compréhension »(3) à l'échelle macrotextuelle d'autre part. Il permet aussi de préciser les relations entre sens et référence, le sens précédant la référence selon F. Rastier (p. 67), de même qu'entre afférence et compléments cognitifs, tout en notant la part déterminante dans le processus d'interprétation, de la « norme sociale, dite aussi socioculturelle ou sociolectale » (p. 73). Les avancées théoriques ne résolvent pas nécessairement les difficultés rencontrées dans la pédagogie qui se donne pour objet ou

(2) Voir l'article « sème » dans R. Galisson et D. Coste (dir.), Dictionnaire de didactique des langues, Paris, Hachette, 1976, p. 485.

(3) Id. p. 302. 
projet de faire émerger une pratique raisonnée de la traduction, là où le processus d'interprétation est « largement intuitif, spontané, effectué sans y penser par le lecteur grâce à sa compétence interprétative » (p. 81). La possibilité de décrire rationnellement « la stratégie et la tactique interprétatives » contribue toutefois fortement à la possibilité de transmettre cette compétence.

\section{Réticulation}

F. Israël dresse quant à lui un panorama du rayonnement de cette théorie en faisant valoir, thèses soutenues à l'ESIT à l'appui, la portée de son extension :

- Géographique et par voie de conséquence linguistique, les langues d'Afrique comme l'ewondo ou d'Asie comme le coréen ayant fait l'objet de travaux spécifiques, ceci pour souligner non pas la spécificité par « paires de langues », mais la prise en compte d'écarts culturels importants ;

- Conceptuelle, puisqu'au fil des années et des travaux de recherche, sont venues s'ajouter à l'interprétation consécutive et de conférence, la traduction littéraire et la confrontation aux courants de pensée les plus divers, qu'il s'agisse de la théorie du langage, de la narratologie, de l'esthétique de la création verbale, de l'approche cognitive de la lecture, de la psychologie génétique ou de la terminologie, liste non exhaustive, mais aussi ;

- Pratique, les différentes modalités ou champs d'application pratiques de la théorie interprétative englobant le doublage et le sous-titrage cinématographiques, l'adaptation pour le théâtre, et la multiplicité des genres textuels : publicité, littérature, pour ne citer que ces deux exemples.

Tous ces travaux témoignent de l'évolution de la théorie interprétative et de son approfondissement, qu'il s'agisse du rapport entre écrit et oral, de celui de la distance ou de la proximité dans l'expression du culturel, de celui des langues, de la révision ou de la complexification de la représentation du processus de traduction, de la prise en compte 
de l'intertextualité ou de la réflexion sur le sens et sur sa bipolarité en tant qu'il est à la fois notionnel et émotionnel, ou du rapport entre sens et forme permettant de faire concevoir la reformulation non plus comme « un travail de copiste » mais comme « un acte d'écriture » (p. 77), et la traduction non plus comme « un sous-produit de l'original mais un texte à part entière » (p. 78). En d'autres termes :

Conçue hors des sentiers universitaires classiques par des professionnels et pour des professionnels, elle bouscule un certain nombre de croyances bien ancrées en affirmant, par exemple que la vraie traduction n'est pas un instrument d'apprentissage ni une opération linguistique mais un acte de compréhension, de communication et d'écriture, qu'elle ne dépend ni du rapport de langues ni du domaine abordé. Autant de principes qui dérangent et ont encore du mal à s'imposer (p. 79).

À l'issue de cette vaste rétrospective, ne serait-on tenté de penser qu'en fait, ce qui a le plus changé au fil des décennies, c'est l'entour de la théorie interprétative, sous la forme des conditions pratiques d'exercice des métiers de la traduction et de l'interprétation, d'une part, et du contexte de formulation - structuralisme et générativisme semblent avoir fait long feu - voire de changement de paradigme, d'autre part ?

Aux yeux des praticiens, il s'agira bien sûr en premier lieu des conditions d'exercice, amenant à tenir compte d'un paramètre souvent mal aimé, celui de la technique, et parfois même à se demander si la compétence dominante en traduction est réellement d'essence langagière. Le rapport du corpus théorique que constitue la théorie interprétative à la réalité qu'il est censé décrire ou dont il est censé rendre compte, s'en trouve modifié, ce qui n'invalide nullement la théorie interprétative, mais oblige ici ou là à des ajustements, à un renouvellement de la description des pratiques et modalités effectives de la traduction, qui, sans être nécessairement les meilleures, n'en sont pas moins dominantes ou en voie de le devenir et se répercutent à leur tour sur la représentation même du processus de traduction.

Par ailleurs, s'il est vrai que dans les années 60, les idées de D. Seleskovitch allaient résolument à l'encontre des idées dominantes (Lederer, t. I, p. 92), le contexte idéologique, pris dans un sens large 
voire panoramique, n'a-t-il pas évolué ? S'il y avait bel et bien lieu de distinguer la traduction des tentatives maladroites ou imparfaites des linguistes d'alors pour en rendre compte - la démonstration a été largement apportée - qu'en est-il aujourd'hui ?

Comme le souligne J.-R. Ladmiral : « la linguistique d'aujourd'hui a fait une place à la sémantique, à une pragmatique de la communication, à une théorie de l'énonciation, à la sociolinguistique et aux interférences du culturel avec le linguistique ... » (t. I, p. 161). À l'étude linguistique de la traduction ont succédé de multiples approches, quitte à emprunter une méthodologie à des disciplines voisines comme la psychologie cognitive, sous la forme de protocoles de verbalisation provoquée, permettant de renouveler les études descriptives de nature empirique et expérimentale précise A. Hurtado-Albir (t. I, p.182-183 et sq.). Il semble également que la dimension sociale d'une opération réputée de communication soit de plus en plus prise en considération, et ce, sous différentes formes, sociolinguistique, socio-psychologique, socio-cognitive, socio-critique, énumération non exhaustive. Parallèlement, la complexification dont s'accompagne l'évolution du champ traductologique, faisant déplorer à certains le surcroît de théorisation, amène à distinguer à l'intérieur de la traductologie bien des territoires distincts, tant par l'objet de leur étude que par leur discours et leur méthodologie.

Si l'ouvrage en trois tomes(4) avait pour objet de rendre hommage à l'œuvre de Seleskovitch, nul doute que le pari a été tenu. La théorie interprétative a traversé les décennies en confortant, contre vents et marées, sa position dans le domaine de la traductologie. Les aléas de sa réception ne seraient-ils imputables, somme toute, qu'à des questions de lecture et de compréhension qui en découlent : hâtives ou lacunaires pour certains, déformantes pour d'autres, interprétatives assurément, partielles et partiales trop souvent ?

(4) Quelques coquilles à signaler : t.1, p. 15 : « en perpétuelle devenir », p. 19 «gode Medal », p. 46 « délibéré ent », t. 2, p. 116, absence de numérotation 3.1 .5 à l'intertitre « Représentation... ». 